\title{
Transplantable functional kidney built on a biocompatible scaffold
}

For the first time, investigators from Massachusetts General Hospital, USA, have successfully created a biocompatible kidney with intact vascular, cortical, and medullary architecture that can produce urine, both in the laboratory and after being transplanted into living animals.

Similar to their previous work in heart and lung regeneration, Song et al. used detergent perfusion to decellularize rat, pig, and human whole-organ kidneys, yielding acellular scaffolds with intact and perfusable vascular, glomerular, and tubular compartments. Using novel seeding technology, the team repopulated these scaffolds with epithelial and endothelial cells and cultured constructs in medium supplemented with

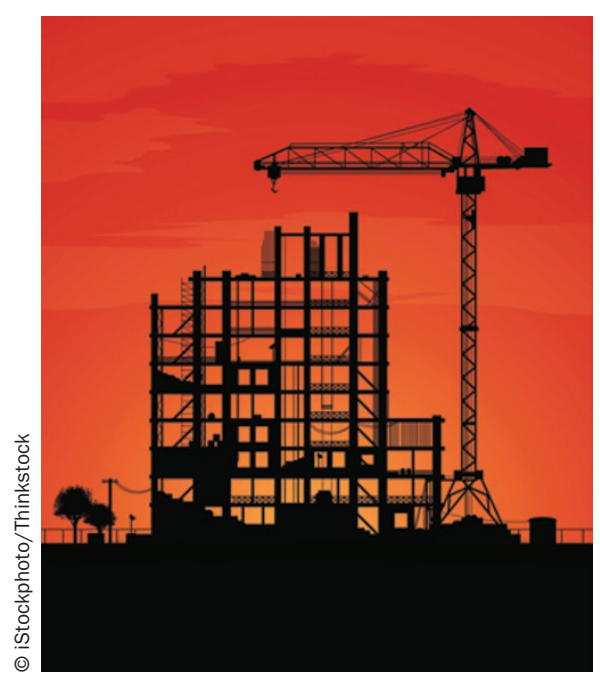

glucocorticoids and catecholamines (in vivo maturation signals) to produce functional transplantable renal grafts.

Importantly, the investigators found that the regenerated rat kidneys functioned in vivo upon orthotopic transplantation into rats that had undergone nephrectomies. Transplanted rats began producing urine shortly after the blood supply was restored, with no evidence of parenchymal bleeding or microvascular clot formation.

"What is unique about this approach is that the native organ's architecture is preserved, so that the resulting graft can be transplanted just like a donor kidney and connected to the recipient's vascular and urinary systems," says Harald Ott, lead researcher of the study and senior author of the Nature Medicine publication.

Although the regenerated kidneys demonstrated reduced creatinine clearance and immature urine filtration and concentration function when compared with cadaveric kidneys, these data are promising.

"The regenerated constructs showed a higher vascular resistance than native kidneys, which is likely a result of immaturity of the vascular bed," says Ott. "We hope that further refinement of seeded cell types, and further in vitro maturation will achieve improved physiologic function."

Ott and his colleagues at Massachusetts General Hospital are currently working with scientists at the Harvard Stem Cell
Institute and the New England Organ Bank (both also in the USA) to develop strategies for scaling scaffold and organ culture technology to human size using cells from patients currently awaiting organ transplants.

"Successful translation of bioengineered kidneys from laboratory bench to bedside will require upscaling of scaffold and organ culture technology to human size, as well as the generation of various cellular phenotypes from patient-derived cells," explains Ott.

If the technology can be applied to human-sized grafts, patients with renal failure who are currently waiting for donor kidneys or who are not suitable candidates for transplantation could be given new organs derived from their own cells, substantially reducing the risk of organ rejection and sparing the patient from a lifetime of immunosuppressive therapy.

"I hope that this study will help to raise interest in the stem cell and developmental biology community and bring the necessary expertise together to identify and expand human kidney progenitor cells," says Ott. "In terms of the timeline for this research, I hope that we will be moving into large animal trials within the next 5 years."

\section{Melanie Clyne}

Original article Song, J. J. et al. Regeneration and experimental orthotopic transplantation of a bioengineered kidney. Nat. Med. doi.10.1038/nm.3154 